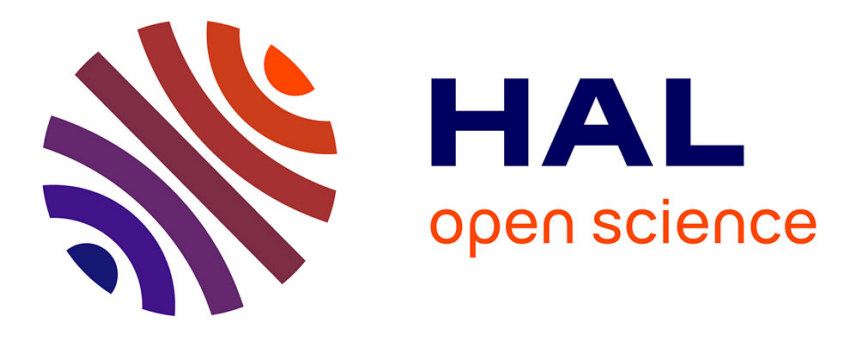

\title{
Nonstatistical behavior of the photoionization of spin-orbit doublets
}

Ralph Püttner, Jessica B Martins, Tatiana Marchenko, Oksana Travnikova, Renaud Guillemin, Loïc Journel, Iyas Ismail, Gildas Goldsztejn, Dimitris Koulentianos, Denis Céolin, et al.

\section{To cite this version:}

Ralph Püttner, Jessica B Martins, Tatiana Marchenko, Oksana Travnikova, Renaud Guillemin, et al.. Nonstatistical behavior of the photoionization of spin-orbit doublets. Journal of Physics B: Atomic, Molecular and Optical Physics, 2021, 54 (8), pp.085001. 10.1088/1361-6455/abef51 . hal-03236214

\section{HAL Id: hal-03236214 https://hal.sorbonne-universite.fr/hal-03236214}

Submitted on 26 May 2021

HAL is a multi-disciplinary open access archive for the deposit and dissemination of scientific research documents, whether they are published or not. The documents may come from teaching and research institutions in France or abroad, or from public or private research centers.
L'archive ouverte pluridisciplinaire HAL, est destinée au dépôt et à la diffusion de documents scientifiques de niveau recherche, publiés ou non, émanant des établissements d'enseignement et de recherche français ou étrangers, des laboratoires publics ou privés. 
PAPER • OPEN ACCESS

Nonstatistical behavior of the photoionization of spin-orbit doublets

To cite this article: Ralph Püttner et al 2021 J. Phys. B: At. Mol. Opt. Phys. 54085001

View the article online for updates and enhancements.

\section{IOP ebooks}

Bringing together innovative digital publishing with leading authors from the global scientific community. Start exploring the collection-download the first chapter of every title for free. 


\title{
Nonstatistical behavior of the photoionization of spin-orbit doublets
}

\author{
Ralph Püttner ${ }^{1, *} \oplus^{\circ}$, Jessica B Martins ${ }^{2}$, Tatiana Marchenko ${ }^{2,3}$ (), \\ Oksana Travnikova ${ }^{2,3}$, Renaud Guillemin ${ }^{23}$, Loïc Journel ${ }^{2,3}$ (), \\ Iyas Ismail $^{2}$, Gildas Goldsztejn ${ }^{2,10}$, Dimitris Koulentianos ${ }^{2,4,11}$, \\ Denis Céolin ${ }^{3}$, Maria Luiza Miranda Rocco ${ }^{5}$, Maria Novella Piancastelli ${ }^{2,6}$ (D), \\ Marc Simon $^{2,3}$, David A Keating ${ }^{7}$, C Rasadi Munasinghe ${ }^{7}$, \\ Pranawa C Deshmukh ${ }^{8,9}$ and Steven T Manson ${ }^{7}$ (1) \\ ${ }^{1}$ Fachbereich Physik, Freie Universität Berlin, Arnimallee 14, D-14195 Berlin, Germany \\ 2 Sorbonne Université, CNRS, Laboratoire de Chimie Physique-Matière et Rayonnement, LCPMR, \\ F-75005 Paris Cedex 05, France \\ 3 Synchrotron SOLEIL, l'Orme des Merisiers, Saint-Aubin, F-91192 Gif-sur-Yvette Cedex, France \\ 4 Department of Physics, University of Gothenburg, Origovägen 6B, SE-412 96 Gothenburg, Sweden \\ 5 Instituto de Quimica, Universidade Federal do Rio de Janeiro, 21.941-909, Rio de Janeiro-RJ, Brazil \\ ${ }^{6}$ Department of Physics and Astronomy, Uppsala University, SE-75120 Uppsala, Sweden \\ 7 Department of Physics and Astronomy, Georgia State University, Atlanta, GA 30303, United States of \\ America \\ ${ }^{8}$ Department of Physics and CAMOST, Indian Institute of Technology Tirupati, Renigunta Road, Tirupati \\ 517506, India \\ 9 Department of Physics, Dayananda Sagar University, Kudlu Gate, Hosur Road, Bengaluru 560114, India \\ E-mail: puettner@physik.fu-berlin.de
}

Received 9 December 2020, revised 1 March 2021

Accepted for publication 16 March 2021

Published 3 May 2021

\section{Abstract}

The photoionization branching ratios of spin-orbit doublets are studied both experimentally and theoretically at energies several keV above threshold. The results show significant relativistic effects for $\operatorname{Ar} 2 p$ in the autoionizing region below the $1 s$ threshold, and large many-body effects for Xe $3 d$ and $4 d$ in the vicinity of the L-shell thresholds. The branching ratios in $\mathrm{Xe}$ are also found to vary significantly over very broad multi-keV energy regions both above and below the inner-shell thresholds. In addition, the Ar $2 p$ study confirms experimentally the decades-old theoretical prediction that the nonresonant branching ratio does not approach the statistical (nonrelativistic) value, and, in fact, progressively diverges from statistical with increasing photon energy.

Keywords: photoionization, interchannel coupling, relativistic effects

(Some figures may appear in colour only in the online journal)

\footnotetext{
10 Permanent address: Université Paris-Saclay, CNRS, Institut des Sciences Moléculaires d’Orsay, 91405, Orsay, France.

${ }^{11}$ Present address: Chemical Sciences and Engineering Division, Argonne National Laboratory, 9700 S Cass Avenue, Lemont, IL 60439, USA.

* Author to whom any correspondence should be addressed.
}

(c) (i) Original content from this work may be used under the terms of the Creative Commons Attribution 4.0 licence. Any further distribution of this work must maintain attribution to the author(s) and the title of the work, journal citation and DOI. 


\section{Introduction}

Photoionization studies have long been important tools in the investigation of the properties of atomic, molecular and condensed matter systems owing to the facts that the coupling of the photon to the target electrons is weak, and the photon disappears after the interaction. Furthermore, the availability of synchrotron light sources of increased brightness in recent years has engendered possibilities for measuring weak processes of quite small cross section over the UV and $\mathrm{x}$-ray energy ranges. In the present work we rely on the quite recent improved instrumentation, both for synchrotron radiation and electron detection, in the tender x-ray regime, 2-10 $\mathrm{keV}[1,2]$.

Among the weak processes that these experimental advances have allowed us to scrutinize are the photoionization of atomic and molecular subshells very far above their thresholds where the cross sections are quite small. In addition, the increased resolution has allowed the measurement of the ratio of the photoionization cross sections of the two components of spin-orbit doublets, subshells split by the spin-orbit interaction. In particular, in the past five years or so, improvements in both the synchrotron source and photoelectron detection in the tender $\mathrm{x}$-ray regime $(2-10 \mathrm{keV})$ have been significant [3] and measurements are now possible that could not be done just a few years ago.

Together these developments make it possible to study experimentally spin-orbit doublet branching ratios, i.e. the ratios of their photoionization cross sections, at energies far above their thresholds. This addresses two long-standing issues concerning these branching ratios. First, for a given $n l$ subshell, it was predicted theoretically that the branching ratio never does approach the statistical ratio, $(l+1) / l$, owing to relativistic effects [4-6]. It was seen that, in the near-threshold region, where the kinetic energy difference of the photoelectrons from the two members of a spin-orbit doublet is significant, and where electron-electron correlations (manybody interactions) are significant, the branching ratio was nonstatistical and even energy-dependent [7, 8]. However, this theoretical prediction concerning the high-energy branching ratio has never been tested experimentally. Second, the question arises of how the branching ratios behave in the vicinity of deeper thresholds, both just below these thresholds, in the region of the infinite series of autoionizing resonances, and just above the thresholds where recent experience has shown significant effects of interchannel coupling [9-11].

To these ends, we have embarked upon a program of experimental and theoretical investigations of these matters, with the photoionization of Ar and Xe being the foci of the present report. In the course of this investigation we found three main results. First, experimental confirmation that, owing to relativistic interactions, the high-energy branching ratio does not approach the statistical ratio, as predicted earlier [4-6] and, in fact, diverge with increasing photon energy from the statistical ratio. Second, the resonances below the deeper ionization thresholds couple differently to the components of the spin-orbit doublets. This effect results in variations of the branching ratio over a range of typically a few eV. Third, interchannel coupling can modify the intensity ratio of the spin-orbit doublets over a wide energy range of about $2 \mathrm{keV}$ which is discussed in the cases of $\mathrm{Xe} 3 d$ and $\mathrm{Xe} 4 d$. We show that these three effects superimpose upon each other in the branching ratios of the spin-orbit doublets that are studied. The experimental and theoretical details are given in the next two sections, respectively, and details of the simulations of the branching ratios in the region of autoionizing resonances are reported in section 4 . Section 5 presents and discusses our results, and the final section presents a summary and conclusions.

\section{Experimental details}

The measurements were performed at the GALAXIES beamline at French national synchrotron facility SOLEIL. The GALAXIES beamline provides monochromatic linearly polarized light, in the photon energy range of $2.3-12 \mathrm{keV}$, with a photon bandwidth between $0.2 \mathrm{eV}$ and $3 \mathrm{eV}$ [1]. The photoelectron spectra were recorded using the hard x-ray photoelectron spectroscopy end-station based on the EW4000 VG Scienta hemispherical analyzer [2]. The spectrometer electrostatic lens system is installed parallel to the polarization vector of the photon beam. The background pressure in the spectrometer chamber, which is proportional to the pressure in the gas cell, was constantly monitored during the measurements and was maintained at $6 \times 10^{-6}$ mbar. The photon flux and the gas pressure were adjusted in a way to keep the count rate sufficiently low, namely less than 1000 counts per sweep for a data acquisition time of $176 \mathrm{~ms}$ at each data point, in order to avoid the detector saturation effects and, therefore, systematic errors for the derived intensity ratios.

In argon, the $2 p^{-1}$ photoelectron spectra, shown in figure 1 , were measured in small energy steps between $3199 \mathrm{eV}$ and $3207 \mathrm{eV}$ photon energy near the Ar $1 s$ ionization threshold located at 3206.3(3) eV [12] and at several more photon energies between $2700 \mathrm{eV}$ and $4000 \mathrm{eV}$. The photon bandwidths were ranging from $0.25 \mathrm{eV}$ at $2700 \mathrm{eV}$ to $0.46 \mathrm{eV}$ at $4000 \mathrm{eV}$.

In xenon, the $3 d^{-1}$ and $4 d^{-1}$ photoelectron spectra were measured between $5092 \mathrm{eV}$ and $5118 \mathrm{eV}$ photon energy near the $\mathrm{Xe} 2 p_{1 / 2}$ ionization threshold located at $5108 \mathrm{eV}$ and between $4772 \mathrm{eV}$ and $4800 \mathrm{eV}$ near the $\mathrm{Xe} 2 p_{3 / 2}$ ionization threshold located at $4787 \mathrm{eV}$ [12]. For Xe $4 d^{-1}$ photoelectron measurements the spectrometer parameters were similar to those used for argon. The photon bandwidth was approximately $0.5 \mathrm{eV}$.

Furthermore, we note that the spectrometer resolution was $80 \mathrm{meV}$ for the $\mathrm{Ar} 2 p$ as well as $\mathrm{Xe} 4 d$ measurements and $180 \mathrm{meV}$ for the $\mathrm{Xe} 3 d$ measurements. However, these values have no influence on the experimental results as long as they are small compared to the spin-orbit splitting of the doublets, which amount to roughly $2 \mathrm{eV}$ for Ar $2 p^{-1}$ and Xe $4 d^{-1}$, and about $12 \mathrm{eV}$ for Xe $3 d^{-1}$.

The kinetic energy was calibrated based on the known energy of Ar LMM normal Auger spectra using the $\mathrm{Ar}$ 


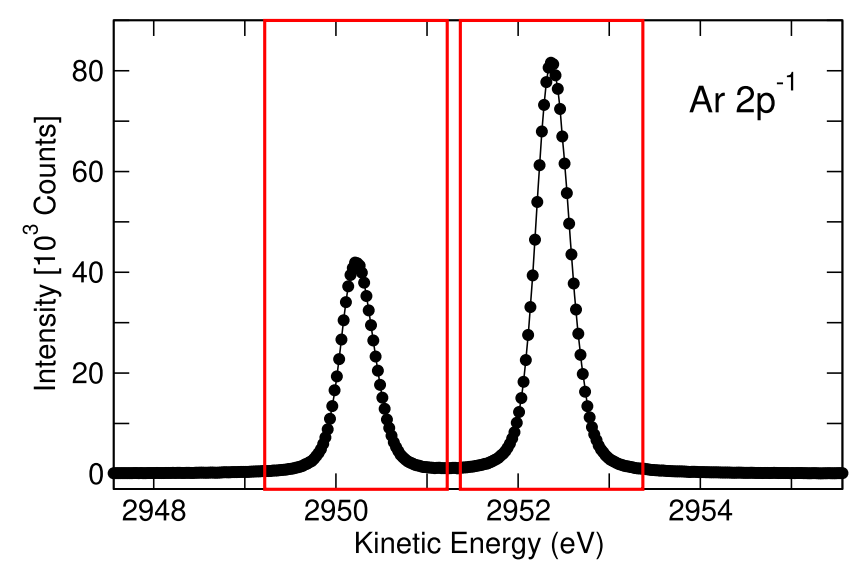

Figure 1. A representative $\operatorname{Ar} 2 p^{-1}$ photoelectron spectrum measured using a photon energy of $h \nu=3201 \mathrm{eV}$. The spectrum was measured with a pass energy of $100 \mathrm{eV}$ and a curved slit with a width of $300 \mu \mathrm{m}$, resulting in a detector resolution of $80 \mathrm{meV}$. The photon bandwidth at $h \nu=3201 \mathrm{eV}$ amounts approximately to 350 $\mathrm{meV}$. The red boxes indicate the energy ranges used for integration to derive the intensity ratios.

$2 p^{-1}$ [13] and $\operatorname{Ar} 3 p^{-2}$ [14] binding energies. For the argon measurements, the photon-energy scale was calibrated using known binding energies of $\operatorname{Ar} 2 p^{-1}$ states [13]. Calibration of the photon energy for xenon measurements relies on the absorption spectrum recorded prior to the photoelectron measurements.

In order to obtain the spin-orbit doublet branching ratios, we subtracted a linear background from the measured spectra and integrated the areas under doublet peaks within a fixed interval around the peak maximum. For this, we chose an interval of $2 \mathrm{eV}$ for $\mathrm{Ar} 2 p^{-1}$ and $\mathrm{Xe} 4 d^{-1}$ photoelectron spectra, and an interval of $4 \mathrm{eV}$ for $\mathrm{Xe} 3 d^{-1}$ photoelectron spectra. As already discussed above, this procedure requires that the instrumental resolution, including photon bandwidth and the detector resolution, is smaller than the splitting. Failing this, the components overlap, and it becomes difficult to obtain accurate values for the ratio. The ratios of the respective integrated areas were plotted as a function of photon energy. The error bars were obtained propagating the experimental statistical uncertainty.

Note that measurements were made, not of cross sections, but of intensities, which are proportional to cross sections. Thus, the ratio of intensities is equal to the ratio of cross sections. Also note, that throughout this publication we use the term 'intensities' to refer to the integrated peak areas obtained in the experiment as discussed above. To obtain the Ar $2 p_{3 / 2} / 2 p_{1 / 2}$ intensity ratio, the counts of each peak were integrated in an interval from $1 \mathrm{eV}$ below the peak maximum to $1 \mathrm{eV}$ above the maximum; these regions are indicated by the red boxes in figure 1. For the spectrum shown, an intensity ratio of 1.930 (3) was derived. Small experimental error bars were required to measure the small variations in the branching ratio predicted by theory. To realize such small error bars $\cong 1670000$ counts in the $2 p_{3 / 2}$ peak and $\cong 865000$ counts in the $2 p_{1 / 2}$ peak were required. To obtain these numbers under the above defined conditions to prevent saturation effects, 80 sweeps with a total acquisition time of about $3 \mathrm{~h}$ was necessary for each photoelectron spectrum which provides one data point for the study of the $\operatorname{Ar} 2 p_{3 / 2} / 2 p_{1 / 2}$ intensity ratio.

Furthermore, the measurements were not made at the magic angle, where the photoelectron intensity is proportional to the partial cross section (PCS) [15], but at $0^{\circ}$, so that angular distribution effects come into play, but only to the extent that the angular distributions of the two components of a spin-orbit doublet differ.

\section{Theoretical details}

The relativistic-random-phase approximation (RRPA) $[16,17]$, along with relativistic multichannel quantum defect theory (RMQDT) [18], has been used to perform the calculations. This methodology is based on the Dirac equation so that relativistic effects, including the spin-orbit interaction, are included on $a b$ initio basis. In addition, both initial-state two-particle two-hole correlations are included in the RRPA, along with final-state correlations in the form of interchannel coupling (configuration interaction in the continuum), which take care of the crucial interactions affecting the branching ratios. The RRPA and RMQDT methodologies are wellknown so they will not be repeated here. Note that the RRPA is gauge-independent, i.e. length and velocity photoionization matrix elements are equal [18].

Calculations were performed for both Ar and Xe. For Ar all relativistic single-excitation channels (including both discrete and continuum) were included:

$$
\begin{aligned}
1 s \rightarrow & p_{1 / 2}, p_{3 / 2} ; 2 s \rightarrow p_{1 / 2}, p_{3 / 2} ; 2 p_{1 / 2} \rightarrow s, d_{3 / 2} \\
& 2 p_{3 / 2} \rightarrow s, d_{3 / 2}, d_{5 / 2} ; 3 s \rightarrow p_{1 / 2}, p_{3 / 2} ; \\
& 3 p_{1 / 2} \rightarrow s, d_{3 / 2} ; 3 p_{3 / 2} \rightarrow s, d_{3 / 2}, d_{5 / 2},
\end{aligned}
$$

for a total of 16 coupled channels. In the case of Xe, all the relativistic single-excitation channels were included except for the $1 s$ channels which were omitted since their binding energy is so much higher than the energies considered that they have negligible influence. Thus, the channels included were:

$$
\begin{gathered}
2 s \rightarrow p_{1 / 2}, p_{3 / 2} ; 2 p_{1 / 2} \rightarrow s, d_{3 / 2} ; 2 p_{3 / 2} \rightarrow s, d_{3 / 2}, d_{5 / 2} ; \\
3 s \rightarrow p_{1 / 2}, p_{3 / 2} ; 3 p_{1 / 2} \rightarrow s, d_{3 / 2} ; 3 p_{3 / 2} \rightarrow s, d_{3 / 2}, d_{5 / 2} ; \\
\quad 3 d_{3 / 2} \rightarrow p_{1 / 2}, p_{3 / 2}, f_{5 / 2} ; 3 d_{5 / 2} \rightarrow p_{3 / 2}, f_{5 / 2}, f_{7 / 2} ; \\
4 s \rightarrow p_{1 / 2}, p_{3 / 2} ; 4 p_{1 / 2} \rightarrow s, d_{3 / 2} ; 4 p_{3 / 2} \rightarrow s, d_{3 / 2}, d_{5 / 2} ; \\
\quad 4 d_{3 / 2} \rightarrow p_{1 / 2}, p_{3 / 2}, f_{5 / 2} ; 4 d_{5 / 2} \rightarrow p_{3 / 2}, f_{5 / 2}, f_{7 / 2} ; \\
5 s \rightarrow p_{1 / 2}, p_{3 / 2} ; 5 p_{1 / 2} \rightarrow s, d_{3 / 2} ; 5 p_{3 / 2} \rightarrow s, d_{3 / 2}, d_{5 / 2},
\end{gathered}
$$

for a total of 40 coupled channels. Technically, the Xe calculation is a truncated RRPA, but as mentioned, the $1 s$ channels have so little influence in the energy region studied that length and velocity results are still virtually identical.

RRPA, as described above, is used to calculate the cross sections in the non-resonant open continuum; RRPA is also used for the input to the RMQDT calculation in which the cross sections in resonance regions are calculated algebraically [18]. 
It should be mentioned that discrete energy scale of the RRPA calculations has essentially no effect on the results because the photoionization parameters are interpolated between the discrete energy points in this region [18]. However, unlike the non-resonant continuum region, where we expect that the RRPA cross sections are excellent representations of the qualitative and quantitative aspects of the cross sections, the situation in the resonance regions is rather different. This is because for inner shells some resonant channels are not included in the RRPA/RMQDT treatment. Specifically, the spectator Auger channels are omitted; only the participator channels are included. For example, in Ar, a $1 s \rightarrow n p$ excitation can lead to an $L$ or $M$-shell electron dropping down to the $1 s$ and transferring the energy to $n p$ electron which emerges as the photoelectron; this is the participator Auger process, since the $n p$ electron participates in the Auger decay, and is included in the RRPA method. However, also possible are $K L L, K L M$ and KMM Auger decays which leave the excited $n p$ electron unchanged, i.e. a spectator. Not only are these the dominant decay processes, but they are not included in RRPA. Thus, while the RRPA/RMQDT methodology obtains the resonance positions quite well, the widths and the shape, $q$ parameters [19], will be quite unreliable owing to the omission. Thus, the widths and shapes of the RRPA/RMQDT theoretical data must be augmented before those can sensibly be compared with experiment. This is discussed in the following section.

It is important to note, however, that these considerations of participator and spectator Auger processes affect the cross sections only in the resonance regions, i.e. for a few $\mathrm{eV}$ below each inner-shell threshold; they have no influence on the nonresonant continuum cross section away from these regions. In addition, the postcollision interaction (PCI) [20] and in particular the electron recapture [21] affects the cross sections of inner shells (here e.g. Ar $1 s$ ) above their respective thresholds. While the PCI does not affect the outer shell cross sections (here e.g. Ar $2 p$ ) directly, owing to interchannel coupling, a change in an inner-shell cross section can affect the outershell cross section over a small region ( $1 \mathrm{eV}$ or so). However, this indirect effect of PCI is likely to be extremely small and not included in the present calculations.

Note also that nondipole effects are not included in the calculation. However, and this is important, at $0^{\circ}$, the lowest-order nondipole effects vanish and the next order are very small indeed. This can be estimated using the nondipole parameters calculated at the relativistic independent particle approximation (central field) which are quite small and pretty much the same for the two members of the spin orbit doublet [22].

\section{Simulations of the branching ratios}

Since we cannot measure the experimental $\sigma\left(n l_{l-1 / 2}\right)$ and $\sigma\left(n l_{l+1 / 2}\right)$ PCSs with sufficiently high accuracy due to variations in the photon flux or gas pressure, we compare the experimental and theoretical ratios $\sigma\left(n l_{l+1 / 2}\right) / \sigma\left(n l_{l-1 / 2}\right)$; actually, as discussed above, the ratio of intensities is measured which gives the same branching ratio since the intensities are

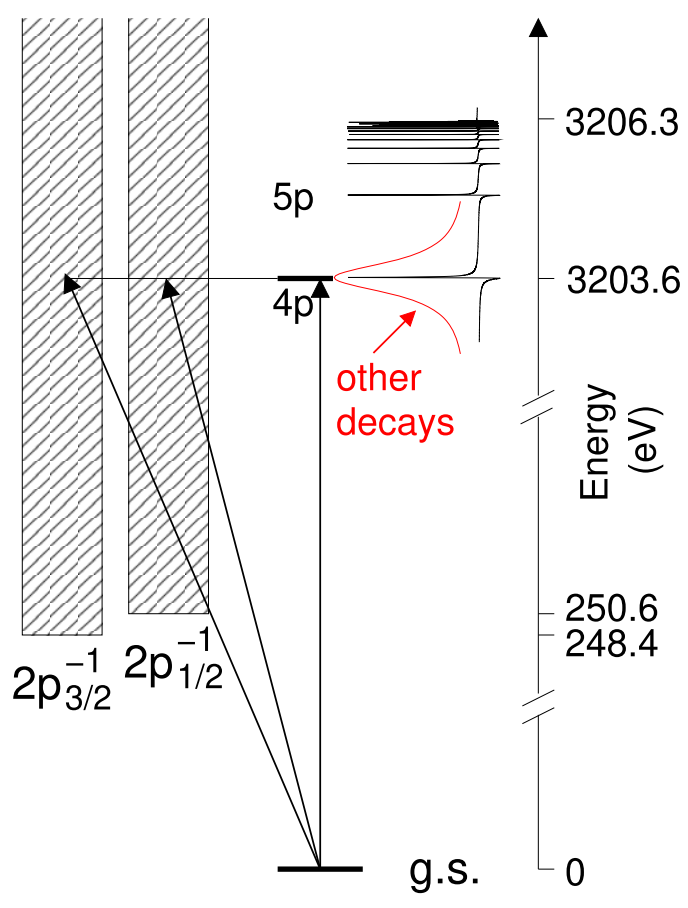

Figure 2. Model for the data analysis. The discrete state $\operatorname{Ar} 1 s^{-1} 4 p$ decays via autoionization to the $2 p$ continua. Different interactions with the $2 p_{3 / 2}$ and $2 p_{1 / 2}$ lead to different effective Fano parameters, $q$, which are the origin for the structures in the ratio close to the Ar $1 s^{-1}$ ionization threshold. The predominant decay of the $\mathrm{Ar}^{-1} s^{-1} 4 p$ to continua other than the $2 p$ channels lead to a Lorentzian-like broadening.

proportional to the cross sections. Far away from inner thresholds the cross sections do not exhibit narrow structures so that the two cross sections can be divided directly. However, close to thresholds the cross sections become more structured and have to be convoluted prior to division. This holds, in particular, for the theoretical $\operatorname{Ar} 2 p_{3 / 2}$ and $2 p_{1 / 2}$ PCSs, which exhibit directly below the $1 s$ ionization threshold very narrow Fano line shapes [19] due to the very small partial decay rate of the participator Auger process, see e.g. figure 3 with corresponding simulations for $q=2.5$. As shown in figure 2 , these structures are caused by the autoionization decay (participator Auger decay) of the $\operatorname{Ar} 1 s^{-1} n p(n \geqslant 4)$ resonances to the $2 p^{-1} \epsilon l$ continuum, the only one taken into account in the calculations. To account for all other decay channels including the dominant KLL Auger decay, the calculated spectra are convoluted with a Lorentzian line shape of $655 \mathrm{meV}$ full width at half maximum representing the $1 s^{-1}$ core-hole lifetime [2]. Finally, the cross sections must be convoluted with a Gaussian of about $350 \mathrm{meV}$ to account for the photon bandwidth. In principle, such a procedure should also be applied to the $\mathrm{Xe}$ calculations. However, for xenon the regions of $2 s^{-1} n p$ as well as $2 p^{-1} n s, n d$ resonances close to the respective threshold are not calculated due to complexity involved with so many open channels; the resulting energy gap renders a convolution impossible.

The ratio derived from the theoretical $\operatorname{Ar} 2 p_{3 / 2}$ and $2 p_{1 / 2}$ PCSs treated with the described convolution procedure showed 
variations directly below the $1 s$ ionization threshold, however, did not agree well with the experimental data, as shown below. This disagreement is due to the omission of spectator Auger channels in the ab initio calculations, as described above, which means that the shapes and widths of the resonances can be quite incorrect.

To overcome the omissions in the $a b$ initio calculations, and to obtain branching ratios that can be compared with the experiment, we performed simulations of the PCSs. Using the formulation of Starace [23] as a starting point, a PCS, in the vicinity of an autoionizing resonance, can be described by

$$
\begin{aligned}
\sigma_{\mathrm{P}}(\epsilon)== & \frac{\sigma_{\mathrm{P}}^{0}}{1+\epsilon^{2}} \times\left(\epsilon^{2}+2 \epsilon\left(q^{\prime} \operatorname{Re} \alpha-\operatorname{Im} \alpha\right)\right. \\
& \left.+\left(1-2 q^{\prime} \operatorname{Im} \alpha-2 \operatorname{Re} \alpha+\left(q^{\prime 2}+1\right)|\alpha|^{2}\right)\right) .
\end{aligned}
$$

Here $\sigma_{\mathrm{P}}(\epsilon)$ is the PCS, $\sigma_{\mathrm{P}}^{0}$ the PCS without the resonances, $q^{\prime}$ the Fano shape parameter, and $\alpha$ the fraction of the dipole amplitude $d$, that passes through the eigenchannel which interacts with the discrete state [23]; note that $\sigma_{\mathrm{P}}(\epsilon)=|d|^{2}$. As shown in the appendix, $\sigma_{\mathrm{P}}(\epsilon)$ can be parameterized using the Fano formula

$$
\sigma(\epsilon)=\sigma_{a} \frac{(q+\epsilon)^{2}}{1+\epsilon^{2}}+\sigma_{b}
$$

with $\sigma_{a}\left(\sigma_{b}\right)$ being the part of the continuum interacting (not interacting) with the Rydberg state. Moreover, $\epsilon=\frac{2\left(E-E_{\mathrm{r}}\right)}{\Gamma}$ with $E$ being the photon energy, $E_{\mathrm{r}}$ the resonance energy of the autoionizing Rydberg state, and $\Gamma$ the lifetime broadening or width. Note that equation (2) is applied to a PCS so that $q$ is not the Fano parameter, but an effective parameter without deeper physical meaning (see appendix for details). Based on equation (2), the PCSs of Ar can be described by

$$
\sigma(E)=\sigma_{a}+\sum_{n=4}^{\infty} \sigma_{a}\left(\frac{\left(q_{n}+\frac{2\left(E-E_{\mathrm{r}, n}\right)}{\Gamma_{n}}\right)^{2}}{1+\left(\frac{2\left(E-E_{\mathrm{r}, n}\right)}{\Gamma_{n}}\right)^{2}}-1\right)+\sigma_{b} .
$$

Here, $n$ is the principal quantum number of the Rydberg states. Moreover, $q_{n}$ is considered to be a constant within an unperturbed Rydberg series; this has been observed, e.g. in the PCSs of doubly excited helium by Menzel et al [24]. Moreover, $\Gamma_{n} \cdot n^{* 3}=\Gamma_{n} \cdot(n-\delta)^{3}=\Gamma_{\mathrm{c}}$ with $\delta$ being the constant quantum defect and $\Gamma_{\mathrm{c}}$ the constant reduced width of the Rydberg series [25]. Finally $E_{\mathrm{r}}=I_{p}-\frac{\mathrm{Ry}}{(n-\delta)^{2}}$ is the energy position of the $n$th resonance with $I_{p}$ being the ionization energy and Ry the Rydberg constant.

In the simulations the Rydberg states up to $n=25$ were taken into account. Moreover, $I_{p}=3206.3 \mathrm{eV}$ [12], $\delta=1.755, \sigma_{a}=1.1$, and $\Gamma_{\mathrm{c}}=115 \mathrm{meV}$ were used. Here, $\delta$ results from the well-known energy position of $3203.6 \mathrm{eV}$ for the $\operatorname{Ar} 1 s^{-1} 4 p$ resonance and $\Gamma_{\mathrm{c}}$ is estimated using our $a b$ initio calculations. For the effective Fano parameter, $q$, the values $-3.5,-2.5,1.5,2,2.5,3,3.5$, and 5 were used; these values result in line shapes similar to those of the ab initio calculations. For the simulations, a step size of $50 \mu \mathrm{eV}$ is chosen. The result for $q=2.5$ is shown as an example in figure 3
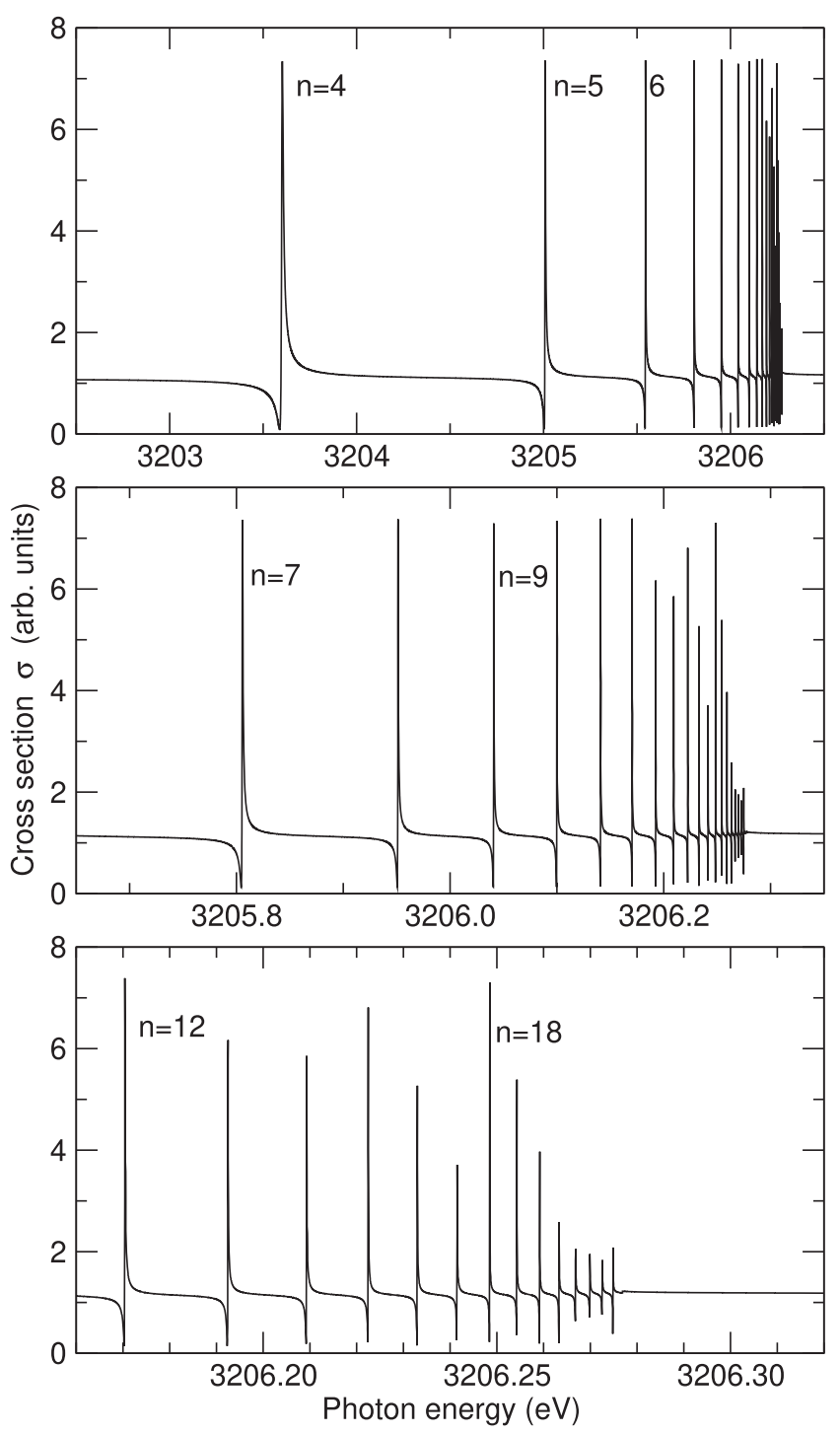

Figure 3. Simulated Rydberg series using $q=2.5$. The middle and the lower panel show details in the high $n$-region. The intensity variations above $n=12$ are due to stepwidths, which are comparable to the linewidths of the Fano-lineshapes. As a result, the peak maxima are not described correctly.

and demonstrates that the small step size allows to obtain equal peak intensities up to $n=12$ as expected for an unperturbed Rydberg series. Moreover, strong deviations from equal peak intensities are not observed below $n=17$, indicating a reasonably small step size. The simulated PCSs are also convoluted with a Gaussian and a Lorentzian as described above. The ratios of the resulting cross sections were derived by using different values for the non-interacting background $\sigma_{b}$.

\section{Results and discussion}

\subsection{Argon}

5.1.1. Nonresonant continuum. The measured $2 p_{3 / 2}: 2 p_{1 / 2}$ branching ratio is shown in figure 4 as a function of photon energy from $2.7 \mathrm{keV}$ to $4.0 \mathrm{keV}$. Both the uncorrected results, i.e. those measured using a detection direction parallel to the 


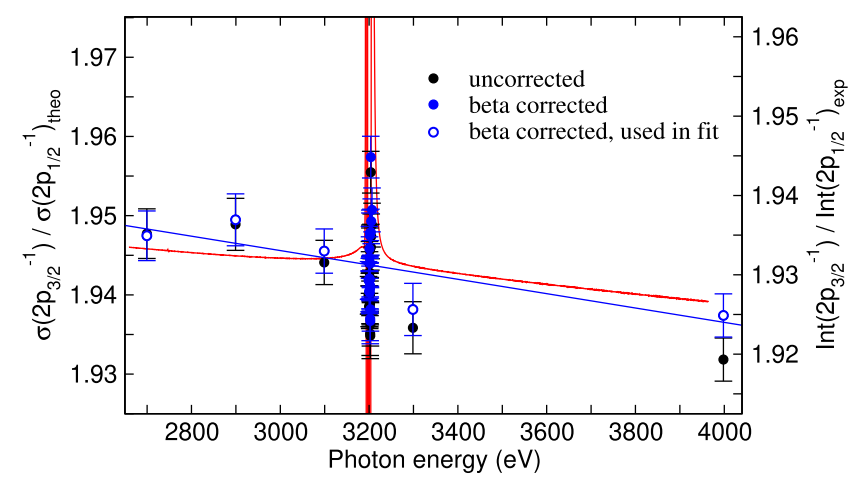

Figure 4. Photoionization branching ratio for Ar $2 p$. Experiment measured at $0^{\circ}$ (black), experiment corrected using theoretical angular distribution parameters, $\beta$ (blue) and theory (red). The blue solid line is a linear fit to the five blue hollow dots. The theoretical data are shifted by $35.3 \mathrm{eV}$ to lower energies in order to match the theoretical and experimental Ar $1 s$ ionization energies.

polarization of the synchrotron radiation and the branching ratios corrected for the angular distribution based on the theoretical results of Derevianko et al [22] (a very small correction) are shown. The structure at about $3.2 \mathrm{keV}$ is due to the opening of the $1 \mathrm{~s}$ ionization channel and will be discussed below. Note that the photon energies are more than an order of magnitude above the $2 p$ thresholds which are $\sim 250 \mathrm{eV}$. The overall trend, in this high-energy region, is for the branching ratio to move away from the statistical value of 2, with increasing energy, in agreement with the earlier theoretical predictions [4-6]. The five off-resonance $\beta$-corrected data points (hollow blue dots) were subject to a fit analysis to a linear function. The solid blue line represents the result of the fit analysis which provided for the ratio a slope of $9(3) \times 10^{-6} / \mathrm{eV}$. This value is a bit larger than the slope of $5.3 \times 10^{-6} / \mathrm{eV}$ derived from the theoretical results based on RRPA (red curve) in the offresonance region. In the off-resonance region above $3.2 \mathrm{keV}$ the theoretical branching ratios are $\approx 0.01$ too high as compared to experiment. In summary, comparison with the theoretical RRPA calculation shows excellent qualitative and pretty good quantitative agreement as well; the slope of the branching ratio moving away from the statistical value is reproduced well by theory. The fact that the branching ratio does not go over to the nonrelativistic limit at high energies, and in fact is moving away from this limit, indicates that relativistic effects must play a part here as suggested by the earlier theoretical results.

Although the general phenomenology was predicted earlier [4-6], to understand in detail what causes this, note that, with increasing energy, the dipole matrix element is generated closer and closer to the nucleus; this can be explained both mathematically and physically. From a mathematical point of view, at the higher energies the continuum wave function becomes more and more oscillatory, resulting in cancellation in the matrix element in the region beyond the first node of the wave function which gets closer and closer to the nucleus as photoelectron energy increases. From a physical standpoint, both energy and momentum must be conserved in the photoionization process. High-energy photoabsorption entails

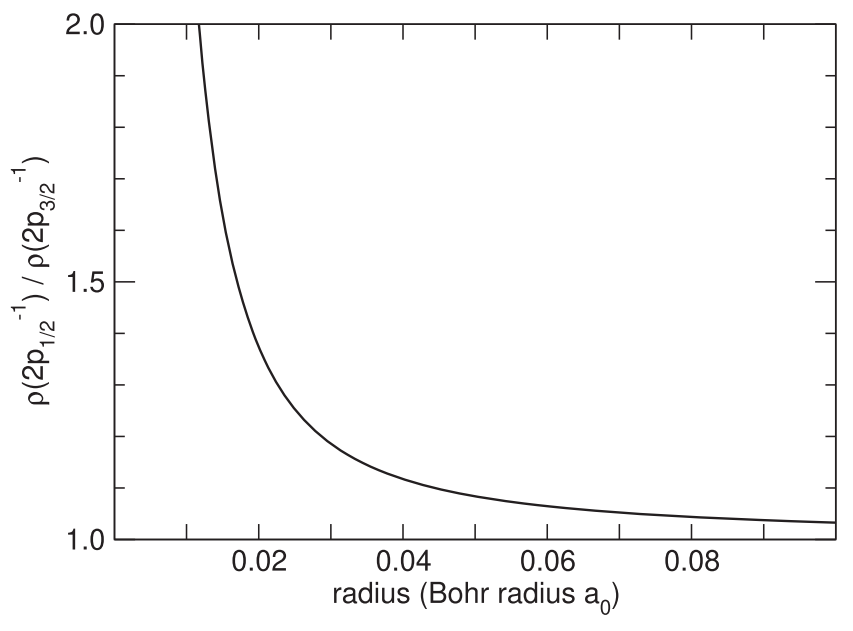

Figure 5. Ratio of the calculated $\operatorname{Ar} \rho\left(2 p_{1 / 2}\right) / \rho\left(2 p_{3 / 2}\right)$ probability densities as a function of the radial coordinate $r$.

a lot of momentum which must be transferred to the residual atom, where most of the mass is at the nucleus. Thus, to take up this momentum, the absorption is most likely to take place near the nucleus, i.e. at small $r$. In any case, at large distances from the nucleus (large $r$ ) the $2 p_{3 / 2}$ and $2 p_{1 / 2}$ wave functions are virtually identical; this is not the case for small $r$ since the behavior of wave functions near the nucleus is determined by $j$, not $l$, as demonstrated by the Dirac equation [26]. Specifically, in this case, the calculated probability density ratio of $2 p_{1 / 2}: 2 p_{3 / 2}$ is shown in figure 5 for small $r$ which demonstrates that this ratio becomes considerably larger than unity at small $r$. This increase in the $2 p_{1 / 2}$ wave function relative to $2 p_{3 / 2}$, as $r$ decreases, is responsible for the anomaly in the branching ratio. Furthermore, it is clear that this effect becomes larger as the photon energy increases, since the matrix element is then generated even closer to the nucleus where the $2 p_{1 / 2}: 2 p_{3 / 2}$ probability density is even larger; as a matter of fact, the ratio diverges as $1 / r^{2}$ as $r \rightarrow 0$ [26]. Specifically, calculations indicate that the important region for the determination of the dipole matrix elements is from the nucleus to $0.3 a_{0}$ at the lowest energy and decreases to $0.2 a_{0}$ at the highest energy. In addition, it is important to note that the Dirac equation also shows the generality of these ideas, i.e. for any non- $s$ subshell holds the above given fact that the probability density ratio $n l_{l-1 / 2}: n l_{l+1 / 2}$ increases as $r$ decreases and diverges as $1 / r^{2}$ as $r \rightarrow 0$.

Although the trends of the experimental and theoretical branching ratios are the same, the absolute values are slightly different; the experimental result is seen to be a bit farther from the statistical ratio than the theoretical branching ratio. There are several possible reasons for this small discrepancy. One possibility is the omission of ionization-plus-excitation (photoionization satellite) channels from the calculation. The $1 s$ cross section, in this energy range, is calculated to be about two orders of magnitude larger than the $2 p$. The $1 s$ ionizationplus-excitation channels are typically smaller by a factor of 10 than the main line (in the case of Ar $1 s$ ionization these contributions were calculated to be $\cong 15 \%$ [27]), so we would expect them to be about an order of magnitude larger than the 


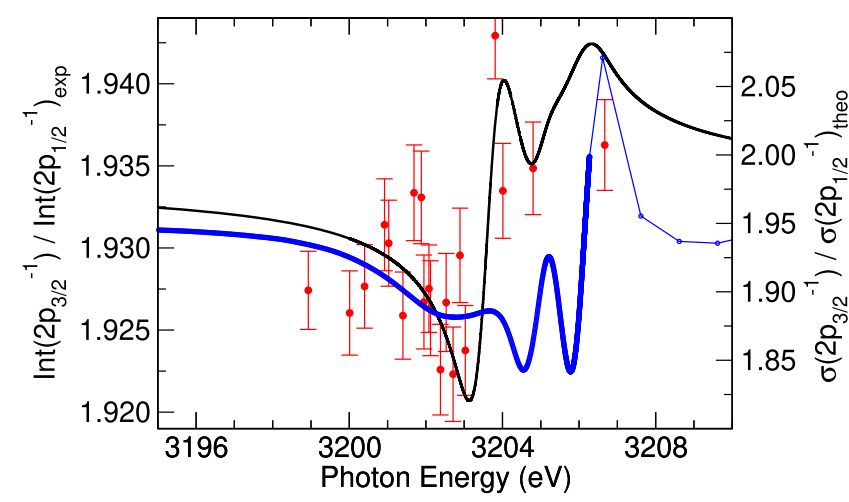

Figure 6. Ar $2 p$ photoionization branching ratio in the region of autoionizing resonances below the $1 s$ threshold. Given are the experimental data (red), the simulated ratio for

$q\left(2 p_{3 / 2}\right)=2.5 / q\left(2 p_{3 / 2}\right)=-2.5$ with $10 \sigma_{a}=\sigma_{b}$, and the ratio of the convoluted $a b$ initio calculations (blue). The transition of the thick to the thin blue line with some data points indicate the ionization threshold since in the step width for the calculations was changed from $10 \mathrm{meV}$ in the resonance region below threshold to $1 \mathrm{eV}$ in the continuum region above threshold. For the experimental data (red) and the simulated ratio (black) the labels on the left axis apply. Note that the ratio obtained from the simulations is shifted by 0.066 to lower values. For the $a b$ initio calculations the labels on the right axis apply; this axis covers a 12 times larger range of branching ratios than the axis on the left.

$2 p$ cross sections. Since it is generally true that interchannel coupling causes channels with small cross section to be altered via the coupling with degenerate (or nearly degenerate) channels with significantly larger cross sections, [9, 28-30] it is expected that the omission of the $1 s$ satellite channels could have the small effect upon the branching ratio that is seen, thereby explaining a possible cause of the small quantitative difference between theory and experiment. In addition, the effect of quadrupole photoionization channels is omitted from the calculation. At photon energies in the 3 to $4 \mathrm{keV}$ range, the quadrupole cross section could be of the order of $1 \%$ of the dipole. And, even though the lowest-order nondipole effects vanish at $0^{\circ}$, if the quadrupole contributes to the spin-orbitsplit cross sections differently, this could have an effect upon the branching ratio.

5.1.2. Branching ratio in the vicinity of the 1 threshold. Figure 6 compares the branching ratios in the vicinity of the $1 s$ threshold obtained from the experimental data (red), from the simulations (black) using $q\left(2 p_{3 / 2}\right)=2.5$ and $q\left(2 p_{1 / 2}\right)=-2.5$ as well as $10 \sigma_{a}=\sigma_{b}$, and from the $a b$ initio calculations (blue). The ratio obtained from the simulations is shifted by 0.066 to lower values and agrees well with the experimental results. Note, that there is no adjustment of the energy. The shift of the simulated results to lower values is necessary since the general decrease of the $\operatorname{Ar} 2 p_{3 / 2}$ to $\operatorname{Ar} 2 p_{1 / 2}$ ratio due to relativistic effects, see figure 4 , is not taken into account in the simulations.

Note that the simulations show a significant increase of the branching ratio at the position of the $\mathrm{Ar} 1 s^{-1} 4 p$ resonance $(3203.6 \mathrm{eV})$. This increase is also clearly present in the experimental data. In detail, the 16 data points lowest in energy, i.e. below $3203.6 \mathrm{eV}$, exhibit the 16 lowest branching ratios, while the 4 data points above this photon energy show the 4 highest ratios. The probability that this match is of statistical origin is only $\left(\begin{array}{c}20 \\ 16\end{array}\right)^{-1}=\left(\frac{20 !}{16 ! 4 !}\right)^{-1} \cong 0.021 \%$.

The ratio derived from the $a b$ initio calculations shows also variations in the energy range of the $\operatorname{Ar} 1 s^{-1} n p$ resonances. The variation with energy is about an order of magnitude larger than the experimentally observed values; note the different scales on the right and left axes of figure 6. Moreover, the theoretically obtained variations in the value do not follow the experimental results qualitatively. Nevertheless, the $a b$ initio calculations clearly demonstrate that the variations in the ratio are caused by the $\mathrm{Ar} 1 s^{-1} n p$ resonances, and this theoretical prediction initiated our experimental study. However, in the course of the experiments it turned out that they are not accurate enough to reproduce the observed variations in the ratio in detail. This evidently means that the spectator Auger decay channels, which were not included in the ab initio calculations, affect the shapes of the resonances in the $\operatorname{Ar} 2 p_{3 / 2}$ and $\operatorname{Ar} 2 p_{1 / 2}$ differently, a purely relativistic effect, thereby strongly affecting the branching ratio in the resonances region. This in addition to increasing the widths of the resonances. Inasmuch as the level of $a b$ initio theory employed is inadequate to deal with the branching ratio in the resonance region, another methodology must be brought to bear to gain some insight into the variation of the ratio just below the Ar $1 s$ threshold that evidently results from differences in the shapes of the resonances in the $2 p_{3 / 2}$ and $2 p_{1 / 2}$ photoionization channels. Thus, as described above, a number of simulations of the PCSs were performed by varying the effective $q$-parameter. Subsequent to convolution, from these PCSs, ratios were derived by combining different $q$-values and using different $\sigma_{b}$ for the noninteracting background. These simulations are presented in detail in the appendix. The most important result is that the overall shape of the ratio depends mainly on the signs of the effective shape parameters $q$ and to a much smaller extent to the exact values of $q$. Three representative results are shown in figure 7 , namely positive $q$-values for $\operatorname{Ar} 2 p_{3 / 2}$ and $2 p_{1 / 2}$ (red solid line), a positive $q$-value or $\operatorname{Ar} 2 p_{3 / 2}$ and a negative $q$-value for $2 p_{1 / 2}$ (blue dashed line), and negative $q$-values for Ar $2 p_{3 / 2}$ and $2 p_{1 / 2}$ (black dash-dotted line). The case of negative $q$-value for Ar $2 p_{3 / 2}$ and a positive $q$-value for $2 p_{1 / 2}$ is not shown but can readily be derived by mirroring the blue dashed line at the straight line with the ratio equal to 2 .

Let us now return to figure 6. It shows one simulated ratio which agrees well with the observed variations caused by the $1 s \rightarrow n p$ resonances. For the shown simulation $q\left(2 p_{3 / 2}\right)=2.5$ and $q\left(2 p_{1 / 2}\right)=-2.5$ was used. Moreover, for both PCSs $\sigma_{b}=10 \sigma_{a}$ is assumed.

The results show that the experimental branching ratio exhibits considerable variation over a small energy range (less than $10 \mathrm{eV}$ ) in the vicinity of the $1 \mathrm{~s}$ threshold. This means that there are significant differences between the resonance structure of the $2 p_{3 / 2}$ and the $2 p_{1 / 2}$ ionization channels. However, the positions (energies) and widths of the $1 s \rightarrow n p$ resonances are properties of the resonances themselves and independent of the particular outgoing channel. The shapes however, the $q$-parameters, are channel-dependent. And, from 


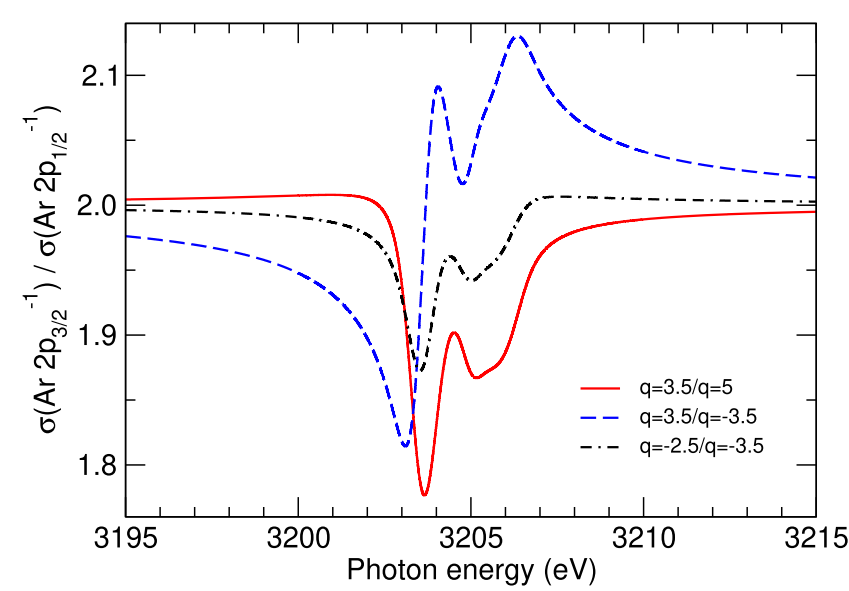

Figure 7. Summary of the obtained ratios. For each combination of $q$, i.e. two positive values (red solid line), one positive and one negative value (blue dashed line) and two negative values (black dash-dotted line) one representative ratio is shown. For all ratios the non-interacting cross section is assumed to be zero.

the experimental results of figure 6, it is evident that the effective $q$-parameters are rather different for the two channels; this is similar to a previous analysis of branching ratios in the neighborhood of a resonance [23]. In particular, the simulations suggest that the effective $q$-parameter for the resonances is positive for the $2 p_{3 / 2}$ PCS and negative for the $2 p_{1 / 2}$ PCS. This difference of resonance shape in different PCSs is similar to the mirroring behavior of resonances discovered earlier in a somewhat different context [31]. Note that other combinations of the sign for the $q$-parameter lead to significantly worse agreement between simulation and experiment. However, we cannot give exact values for the two channels since the differences of our simulations are smaller than the experimental error bars. For example, a simulation with $q\left(2 p_{3 / 2}\right)=3.5$ and $q\left(2 p_{1 / 2}\right)=-3.5$ as well as $\sigma_{b}=15 \sigma_{a}$ fits equally to our experimental results as the simulation shown in figure 6 . In any case, the $1 s^{-1} n p$ resonances clearly couple differently to the $2 p_{3 / 2}$ and the $2 p_{1 / 2}$ continua, a purely relativistic effect. In detail, there are at least two reasons. First, the autoionization matrix element $\left\langle 1 s^{-1} n p|1 / r| 2 p_{j}^{-1} \epsilon l\right\rangle$ (with direct and exchange terms) can differ for $2 p_{3 / 2}$ and the $2 p_{1 / 2}$ due to the different radial distributions of their respective wave functions, particularly near the nucleus. Second, for the decay of the $1 s \rightarrow n p$ resonances to the $2 p_{3 / 2}$ hole, $\epsilon s_{1 / 2}$, $\epsilon d_{3 / 2}$, and $\epsilon d_{5 / 2}$ continua are possible, while for a $2 p_{1 / 2}$ hole only $\epsilon s_{1 / 2}$ and $\epsilon d_{3 / 2}$ are allowed by angular momentum and parity conservation constraints. Furthermore, the differences are clearly important enough that they are still evident even after the convolution of the model theoretical data with the experimental resolution.

Finally note that the third effect that influences the branching ratio, the interchannel coupling, is very small in this case. It leads in the photon energy range of 3000 to $3150 \mathrm{eV}$ to a very small increase of the branching ratio by less than 0.003 . This finding of a small influence on the branching ratio seems to be typical for interchannel coupling when an $s$-shell threshold is involved, as seen below for Xe around the $2 s$ threshold.

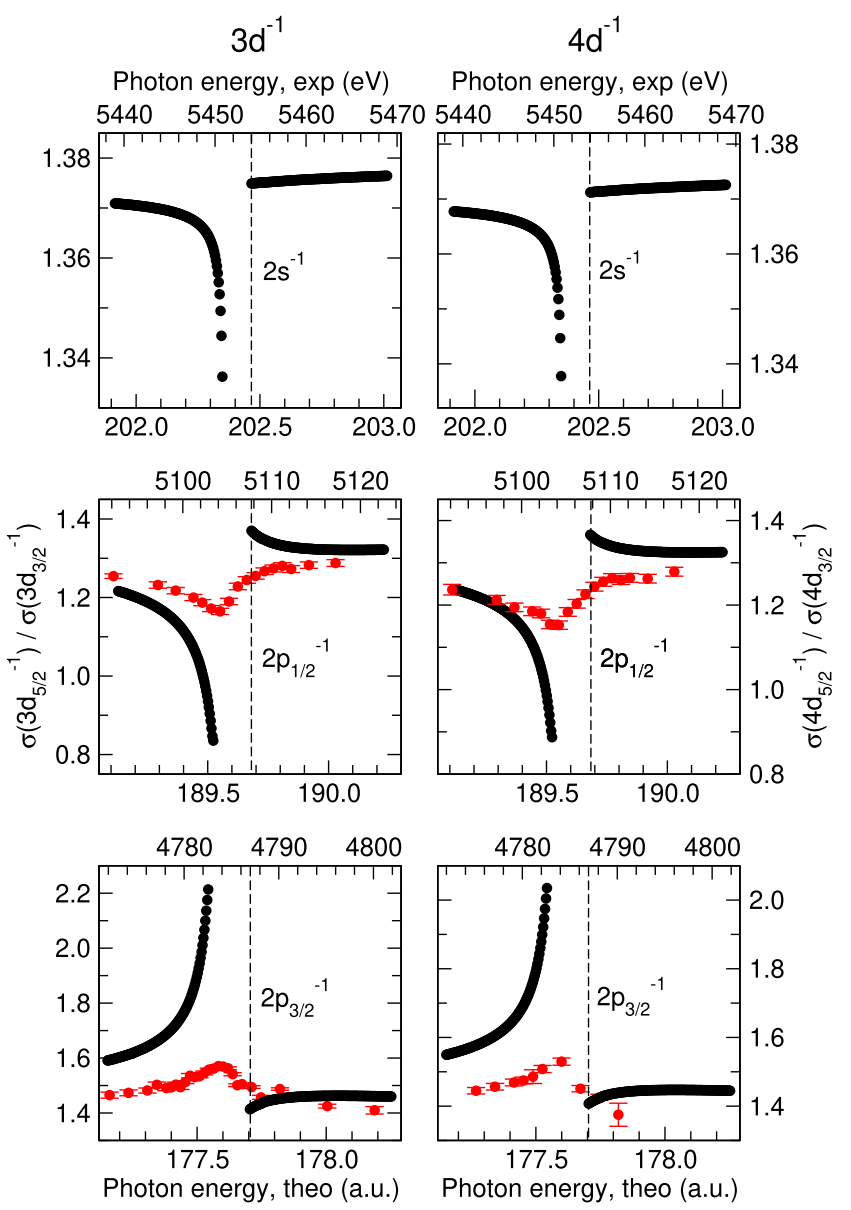

Figure 8. Xe $3 d$ and $4 d$ photoionization branching ratio in the vicinity of the $n=2$ thresholds. Experiment (red), theory (black). The tailing up or down in the theoretical results just below each threshold indicates the beginning of the first resonance of the Rydberg series which is averaged over in the experiment. The experimental and theoretical energy scales are shifted relative to each other so that the respective ionization energies are located at the dashed vertical lines.

\section{2. $\mathrm{Xe}$}

For Xe we find experimentally that the $3 d$ and $4 d$ branching ratios are influenced over a small energy range of a few $\mathrm{eV}$ by the resonances below the $2 p_{3 / 2}$ and $2 p_{1 / 2}$ thresholds, as well as over a wide range of several $\mathrm{keV}$ by strong interchannel coupling. The relativistic effect on the wavefunctions causing an increasing deviation of the branching ratio from the statistical value with increasing photon energy is, however, shown only theoretically, since for xenon it is juxtaposed with strong interchannel coupling that complicates the situation.

Experimental data have been obtained for the $3 d$ and $4 d$ branching ratios in the vicinity of the $2 p_{3 / 2}$ and $2 p_{1 / 2}$ thresholds and the results are shown in red in figure 8 , together with theoretical results in black, which also include the $2 s$ threshold. Note that there has been no modification of the calculated results for experimental conditions since the resonances close to threshold are not calculated; see above. Because of 


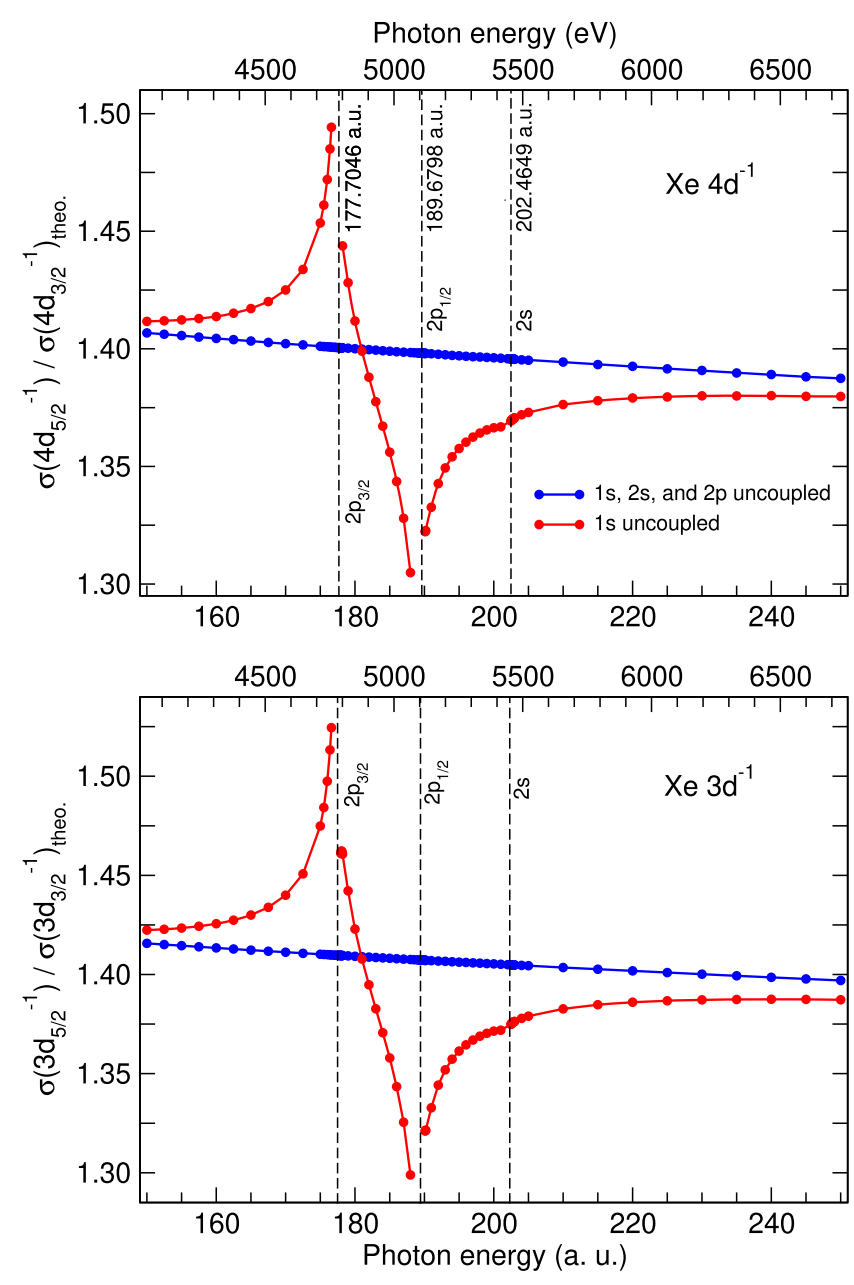

Figure 9. Calculated photoionization branching ratios for $\mathrm{Xe} 3 d$ and $4 d$. Full calculation (red), calculation omitting coupling with $n=2$ channels (blue). The energies in $\mathrm{eV}$ are obtained by multiplying the theoretical values by 0.99 Hartree (a.u.) since in this way the Xe $2 s$ and Xe $2 p$ ionization energies are reproduced well.

this we would not expect complete agreement. Overall, however, the agreement is pretty good; the experimental trends are certainly reproduced. Below each threshold, the beginning of the lowest-energy resonance is seen in the calculated results; these are completely washed out in the experimental results owing to the averaging implicit in the measurements, which is caused by the Xe $2 p$ lifetimes that result in an energy width of $\approx 3 \mathrm{eV}$ [32] and the photon bandwidth of $\approx 0.5 \mathrm{eV}$. The trends below the thresholds are well-reproduced by the calculation showing a rise at the $2 p_{3 / 2}$ threshold and a dip at the $2 p_{1 / 2}$ threshold; evidently the resonances have rather different shapes in the two cases.

It is of interest to note that the experimental and theoretical $3 d$ and $4 d$ branching ratios in the vicinity of the different $n=2$ thresholds are almost the same, both as to absolute values and variation with energy. This occurs because, as mentioned in connection with Ar, the matrix elements for photoionization for both $3 d$ and $4 d$ are generated primarily quite close to the nucleus, at very small $r$. And, for small $r$, although the ratio of the doublet wave functions is independent of angular momentum, the actual form of the wave functions is dependent upon

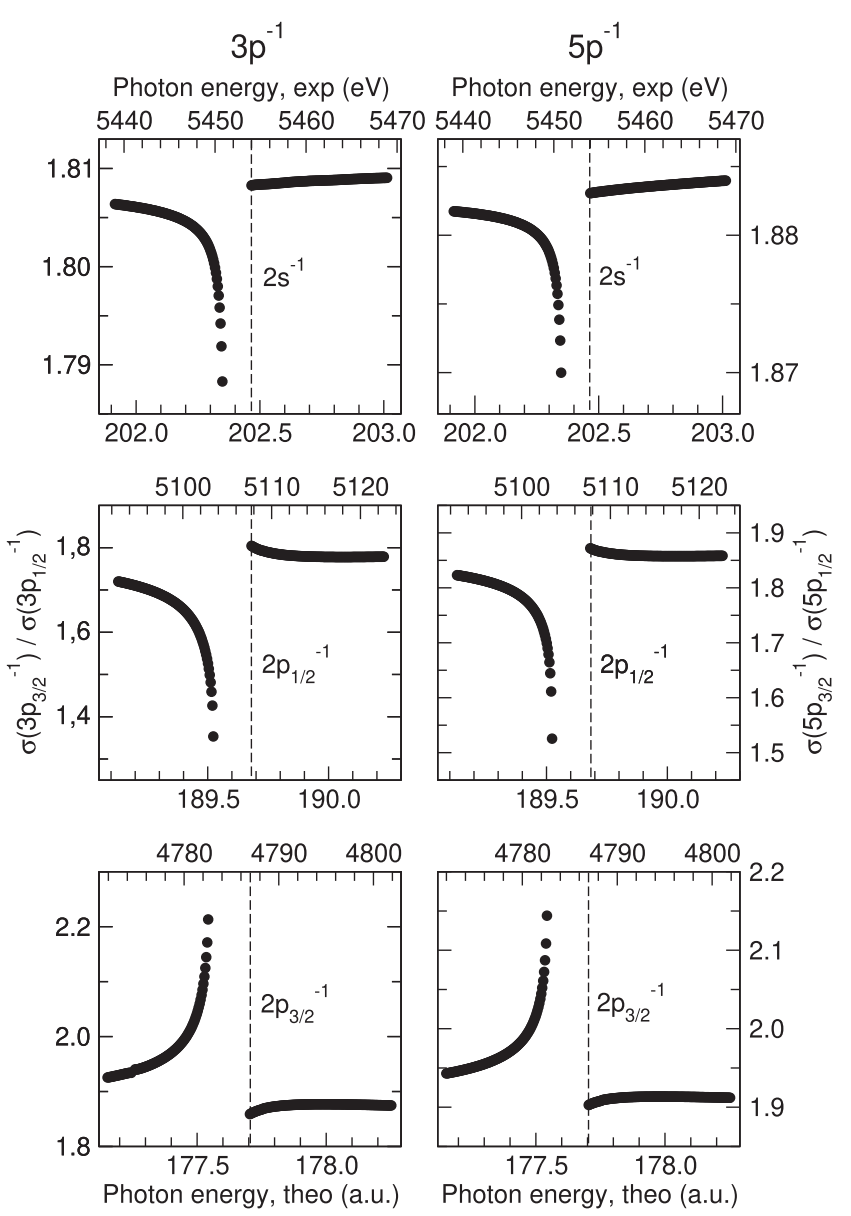

Figure 10. Xe $3 p$ and $5 p$ photoionization branching ratios in the vicinity of the $n=2$ thresholds. The tailing up or down in the theoretical results just below each threshold indicates the beginning of the first resonance of the Rydberg series. For details of energy scales see figure 8 .

angular momentum [26]; the only difference between $3 d$ and $4 d$ being a normalization factor. But, since we are looking at branching ratios, the normalization factor cancels out. Furthermore, as discussed above, the small $r$ ratio of the charge densities of a spin-orbit doublet is independent of angular momentum. Hence both $3 d$ and $4 d$ yield essentially the same branching ratios at these high energies.

Note further that, in the vicinity of the $2 p_{3 / 2}$ threshold, both the $3 d$ and $4 d$ branching ratios are about 1.45; around the higher-energy $2 p_{1 / 2}$ threshold, the branching ratios are about 1.3 , i.e. further away from the statistical ratio of 1.5 ; this agrees with the trend seen in Ar. However, the branching ratio is seen to be in the 1.37 range in the vicinity of the $2 s$ threshold. In other words, the branching ratio is closer to the statistical ratio near the higher-energy $2 s$ threshold, and further from the statistical ratio near the lower-energy $2 p_{1 / 2}$ threshold, thereby appearing to contradict the notion that, asymptotically, the branching ratio decreases with energy owing to the behavior of the discrete wave functions close to the nucleus.

To understand this phenomenon, and to show that it is caused by interchannel coupling, we have calculated branching ratios over a broad energy range, from 150-250 a.u. 
$(\sim 2.7 \mathrm{keV})$ and the results are shown in figure 9 for both the $3 d$ and $4 d$ cases. To further elucidate, the calculations were done both with and without coupling with the $n=2$ subshells. Without those couplings, the expected smooth decreasing branching ratio is seen, resulting from the relativistic behavior of the discrete wave functions near the nucleus. Including the coupling with these $n=2$ photoionization channels is seen to change the picture dramatically which is obviously caused by the interchannel coupling. The coupling causes a sharp drop in the ratios between the $2 p_{3 / 2}$ and $2 p_{1 / 2}$ thresholds, and a gradual increase above the $2 p_{1 / 2}$ and $2 s$ thresholds. This solves the puzzle as to why the branching ratios near the $2 s$ threshold are larger than those near $2 p_{1 / 2}$.

Figure 9 also shows that the interchannel coupling effects of the $2 s$ channels are far smaller than the effects of the $2 p$ channels on the $n d$ branching ratios. This result is in keeping with the Ar case above. Apparently interchannel coupling of outer-shells with inner-shell $n s$ cross sections is much weaker than the coupling with inner $n p$ channels. But a definitive explanation for why this occurs will require further studies. In addition, it is clear that interchannel coupling affects the branching ratios over a huge energy range, and not just near the thresholds; in this case over an energy range of several $\mathrm{keV}$. This demonstrates how important interchannel coupling is over a broad range both below and above inner-shell thresholds. This also shows the power of using the truncated RRPA calculation focus upon the dependence of the cross sections to particular interchannel couplings.

For completeness, the theoretical branching ratios for $\mathrm{Xe}$ $3 p$ and $5 p$ photoionization are shown in figure 10 , even though there is no experimental data for these cases. The results are similar to the $n d$ results. It is however clear that the branching ratios, at least in the threshold region, are rather further away from the statistical value of 2 than was the case for Ar; the branching ratios above each of the thresholds are about 1.9 or below. This is not surprising as we are significantly higher in energy for $\mathrm{Xe}$, and $\mathrm{Xe}$, being higher $Z$, is quite a bit more relativistic than Ar. There is an important difference from the $n d$ case. The $3 p$ and $5 p$ branching ratios are no longer essentially the same (note the different scales of the $3 p$ and $5 p$ plots) like the $3 d$ and $4 d$ ratios are, i.e. in this case the ratios depend upon the principal quantum number, $n$, as well. This is because one of the subshells involved is the $5 p$, the valence subshell of $\mathrm{Xe}$. Although the explanation given for the $n d$ 's remains true, as far as the contribution to the matrix elements near the nucleus, so little of the amplitude of the $5 p$ wave functions lies in this region, that even though the contributions of the outer parts of the $5 p$ wave functions to the matrix elements are mostly canceled out owing to the oscillatory nature of the high-energy continuum wave functions, the little bit that is not canceled matters. If this explanation is correct, going to still higher energies, should bring the ratios for $3 p$ and $5 p$ into better agreement.

\section{Summary and conclusions}

The branching ratios of the photoionization cross sections of spin-orbit doublets well above threshold in Ar and Xe were investigated in a combined experimental and theoretical study. The experimental results on Ar over a broad range of photon energies confirmed earlier theoretical predictions [4-6] that, at the higher energies, the branching ratios were found to diverge from the statistical ratios as the energy increased, rather than approaching the statistical (nonrelativistic) value. This results from the differences in the small- $r$ behavior of the $j=l \pm 1 / 2$ wave functions of the doublet; the $j=l-1 / 2$ wave function dominates near the nucleus, a region that becomes more and more important to the determination of the photoionization matrix element as energy increases. This behavior was found for $\operatorname{Ar} 2 p$ and $\mathrm{Xe} 3 d$ and $4 d$ but it is by no means restricted to those cases. Since the Dirac equation shows that, close to the nucleus, this $j=l-1 / 2$ wave-function dominance, compared to $j=l+1 / 2$, is general, this phenomenon must apply to the high-energy branching ratios of all doublets of all atoms. And, it will apply to molecules as well, since, near the nuclei, molecular wave functions are very atomic-like. Thus, the phenomenon is completely general.

It was found earlier that interchannel coupling significantly affects the branching ratios in the vicinity of inner-shell thresholds [10], but the present investigation has uncovered new aspects of the phenomenon. First, it was shown that the effects of inner shells upon the branching ratios were by no means localized to the energies of the thresholds; for the cases of Xe $3 d$ and $4 d$ the interchannel effects around the $n=2$ thresholds were calculated to significantly affect the branching ratios over a very wide range of energies, about $3 \mathrm{keV}$, both above and below the thresholds. Second, the rapid variation of the $\mathrm{Xe}$ branching ratios with energy, particularly between the $2 p_{3 / 2}$ and $2 p_{1 / 2}$ thresholds, shows that the relativistic interchannel coupling matrix elements are strongly dependent upon energy. Third, the present study measured the Ar $2 p$ branching ratio in the resonance region just below the $1 s$ threshold as well as the $\mathrm{Xe} 3 d$ and $4 d$ branching ratios in the resonance region below the $2 p$ thresholds and found strong variations over that small energy range; a previous experiment looked at photoionization in a condensed matter environment, where the Rydberg structure and the dynamics of low-energy photoelectrons are rather different [10]. It is suggested that further studies of branching ratios in inner-shell resonance regions be conducted to further elucidate the physics of what goes on there.

As a final remark, we reiterate the point that the measurements detailed herein were only possible owing to recent advance in experimental technology. It is hoped that this work will stimulate further advances both in experimental techniques and in theoretical methodology so as to be able to treat these phenomena in still greater detail. 

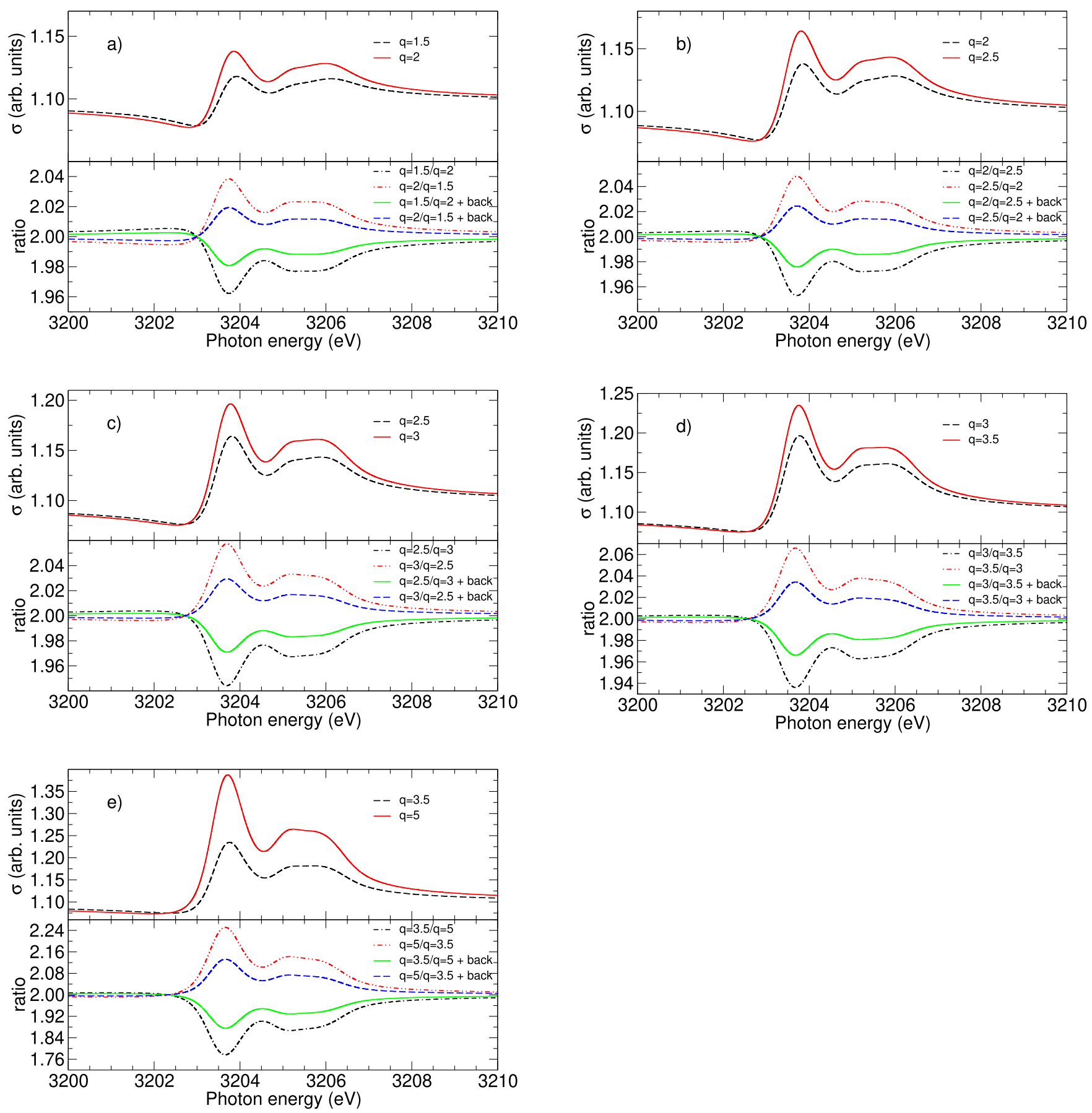

Figure 11. The simulated results using (a) $q=1.5$ and $q=2$, (b) $q=2$ and $q=2.5$, (c) $q=2.5$ and $q=3$, (b) $q=3$ and $q=3.5$, and (e) $q=3.5$ and $q=5$. The upper panels show the simulated Rydberg series after convolution and the lower panels the resulting ratios. For the ratios indicated with 'back' $\sigma_{a}=\sigma_{b}$ is used. For the other two curves $\sigma_{b}=0$ (i.e. no non-interacting background) is applied.

\section{Acknowledgments}

Experiments were performed on the GALAXIES beamline at SOLEIL Synchrotron, France (Proposal Nos. 20150921, 99160189 and 99180102). We are grateful to the SOLEIL staff for smoothly running the facility. TM and JM acknowledge financial support from the French Agence Nationale de la Recherche (ANR) through the ATTOMEMUCHO project (ANR-16-CE30-0001). The work of STM was supported by the US Department of Energy, Office of Science, Basic Energy
Sciences under Award No. DE-FG02-03ER15428. Discussions with Anthony Starace, University of Nebraska are gratefully acknowledged. DK wishes to acknowledge financial support from LabEx MiChem, France, as well as the Swedish Research Council (VR) and Knut and Alice Wallenberg Foundation, Sweden.

\section{Data availability statement}

The data that support the findings of this study are available upon reasonable request from the authors. 


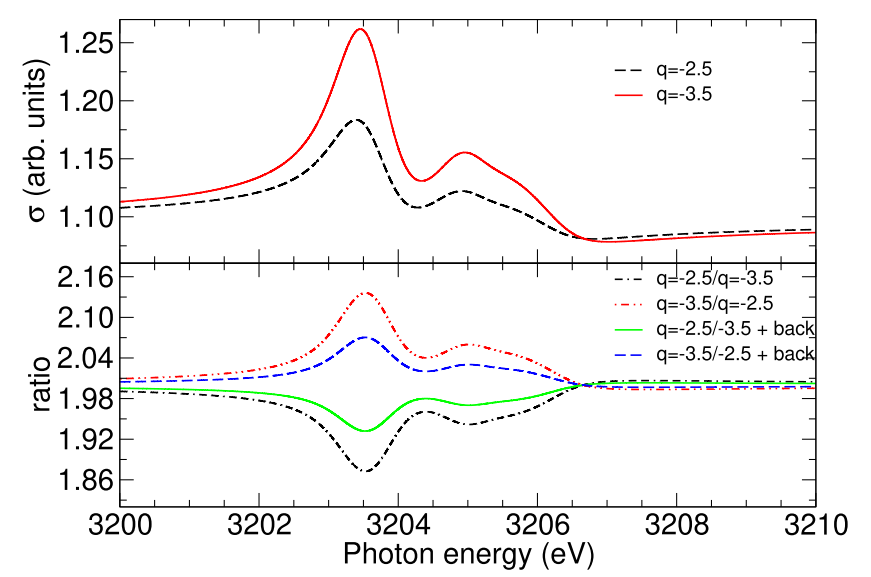

Figure 12. Simulated Rydberg series using $q=-2.5$ and $q=-3.5$ as well as the resulted ratios. For details, see figure 11.
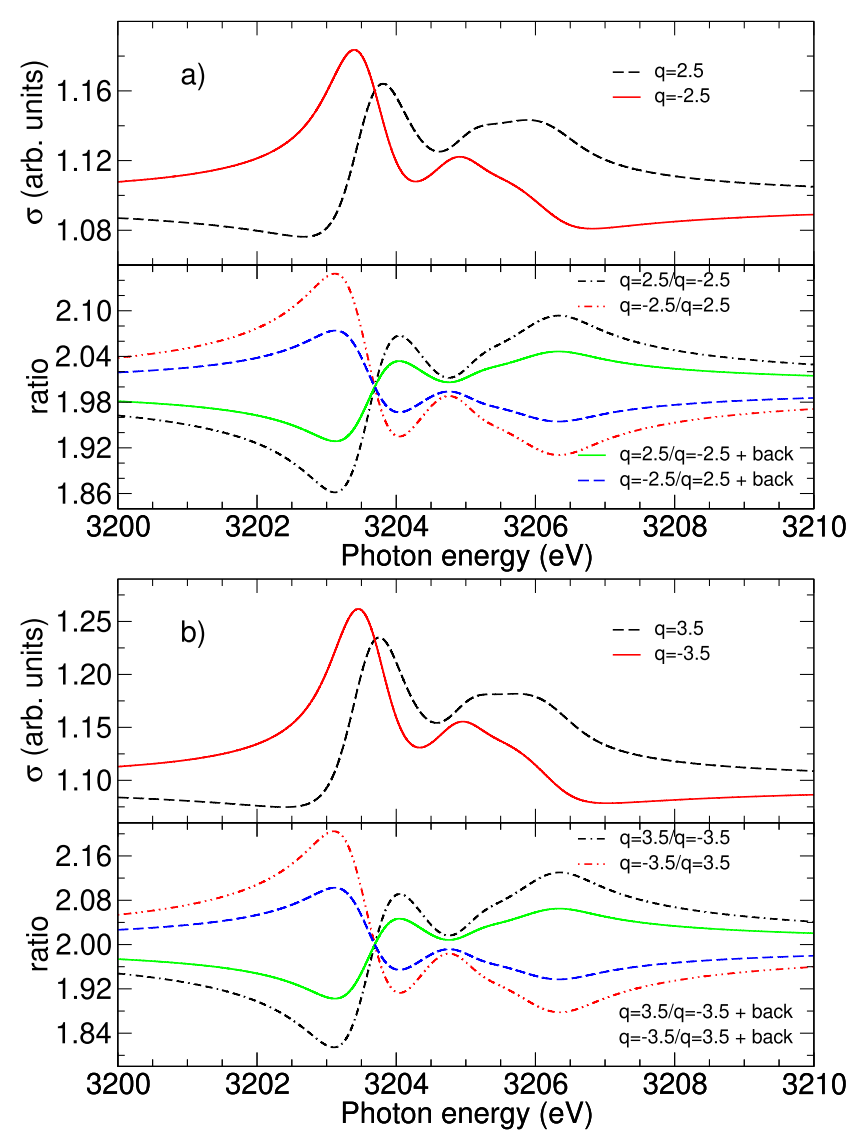

Figure 13. The simulated results using (a) $q=2.5$ and $q=-2.5$, (b) $q=3.5$ and $q=-3.5$. For details, see figure 11 .

\section{Appendix A. Parametrization of partial cross sections}

According to Starace [23], a PCS with an autoionizing resonance can be described with

$$
\begin{aligned}
\sigma_{\mathrm{P}}(\epsilon)= & \frac{\sigma_{\mathrm{P}}^{0}}{1+\epsilon^{2}} \times\left(\epsilon^{2}+2 \epsilon\left(q^{\prime} \operatorname{Re} \alpha-\operatorname{Im} \alpha\right)\right. \\
& \left.+\left(1-2 q^{\prime} \operatorname{Im} \alpha-2 \operatorname{Re} \alpha+\left(q^{\prime 2}+1\right)|\alpha|^{2}\right)\right) .
\end{aligned}
$$

Here $\sigma_{\mathrm{P}}$ is the PCS, $\sigma_{\mathrm{P}}^{0}$ is the PCS without the resonances, $q^{\prime}$ the Fano parameter, and $\alpha$ the fraction of the dipole amplitude $\sigma_{\mathrm{P}}(\epsilon)$, that passes through the eigenchannel which interacts with the discrete state.

We will show that $\sigma_{\mathrm{P}}(\epsilon)$ can be parameterized using the Fano formula for autoionization [19], i.e. it can be described by

$$
\sigma(\epsilon)=\sigma_{a} \frac{(q+\epsilon)^{2}}{1+\epsilon^{2}}+\sigma_{b}
$$

with $\sigma_{a}\left(\sigma_{b}\right)$ being the part of the continuum interacting (not interacting) with the Rydberg states. For this we set in equation (4) $\sigma_{\mathrm{P}}^{0}=1$ for simplicity (this just means that we are taking $\sigma_{\mathrm{P}}$ in units of $\sigma_{\mathrm{P}}^{0}$ ) and define $C_{1}=q^{\prime} \operatorname{Re} \alpha-\operatorname{Im} \alpha$ as well as $C_{2}=1-2 q^{\prime} \operatorname{Im} \alpha-2 \operatorname{Re} \alpha+(q+1)|\alpha|^{2}$. In this way we obtain

$$
\begin{aligned}
\sigma_{\mathrm{P}}(\epsilon) & =\frac{\epsilon^{2}+2 \epsilon C_{1}+C_{2}}{1+\epsilon^{2}} \\
& =\frac{A \epsilon^{2}+A}{1+\epsilon^{2}}+\frac{(1-A) \epsilon^{2}+2 \epsilon C_{1}+C_{2}-A}{1+\epsilon^{2}} \\
& =A+(1-A) \frac{\epsilon^{2}+2 \epsilon \frac{C_{1}}{1-A}+\frac{C_{2}-A}{1-A}}{1+\epsilon^{2}} \\
& =A+(1-A) \frac{\epsilon^{2}+2 \epsilon q+q^{2}}{1+\epsilon^{2}} \\
& =A+(1-A) \frac{(\epsilon+q)^{2}}{1+\epsilon^{2}}
\end{aligned}
$$

with $q=\frac{C_{1}}{1-A}$ and $q^{2}=\frac{C_{2}-A}{1-A}$. From this it follows that $q^{2}=\frac{C_{1}^{2}}{(1-A)^{2}}=\frac{C_{2}-A}{1-A}$ and $A=-\frac{1+C_{2}}{2} \pm \sqrt{\left(\frac{1-C_{2}}{2}\right)^{2}+C_{1}^{2}}$, i.e. the argument of the square root is always positive so that a solution for $A$ exists independent of the specific $C_{1}$ and $C_{2}$. As a consequence the Fano-like parameterization given in equation (6) exists for all cases of $C_{1}$ and $C_{2}$. Note that $q$ in equation (6) is an effective parameter without deeper physical meaning, contrary to the case originally described by Fano with $\sigma$ being the total ionization cross section.

Obviously, $A$ can be interpreted as the non-interacting background $\sigma_{b}$ and $1-A$ as interacting background $\sigma_{a}$ so that we obtain equation (5). Since these cross sections are physical quantities which cannot be negative we obtain $0 \leqslant A \leqslant 1$ as a requirement. As a result, there are restrictions for the values of $C_{1}$ and $C_{2}$. This is not surprising since both quantities are formed by $\alpha$ and $q^{\prime}$ so that they are not fully independent.

\section{Appendix B. Detailed results of the simulations}

In this part of the appendix the simulations of the partial cross sections and ratios are presented. As can be seen in figure 2, the autoionization cross section in figure 3 represents the excitation into a discrete state which decays via autoionization into the $2 p$ continua. Actually, the interaction with the $2 p_{3 / 2}$ and the $2 p_{1 / 2}$ continua is different and leads to different effective Fano parameters, $q$, for the decay to each continuum. As we show below, these differences in the interaction lead to variations in 
the $\operatorname{Ar} 2 p_{3 / 2}^{-1}$ and $2 p_{1 / 2}^{-1}$ intensity ratio as a function of the photon energy, see figures $11-13$. Note that identical $q$ result in identical PCS's and constant ratios.

As described above, the PCSs obtained with equation (3) are convoluted with a Lorentzian of $655 \mathrm{meV}$ width to simulate the major decay channels and a Gaussian of $350 \mathrm{meV}$ width to account for the photon bandwidth. The convoluted PCSs with positive effective $q$ are shown in figures 11(a)-(e). The main difference is that the intensity of the lines increases with $q$. From these convoluted cross sections the ratios are derived.

Due to the similar cross sections, the ratios obtained are also quite similar. The main differences are the amplitudes of the ratios. Note that in figures 11(a)-(d), where the two cross sections (and ratios) are for various values of $q$ and $q$ +0.5 in each, the ratios increase with increasing $q$. Applying an additional non-interacting background $\sigma_{b}$ leads to an decrease of the amplitudes in the ratio. Moreover, the peak maximum of the $\mathrm{Ar} 1 s^{-1} 4 p$ resonance (and for all other unresolved Ar $1 s^{-1} n p$ resonances) is above the value of the resonance position in the photoabsorption spectrum of $3203.6 \mathrm{eV}$. This also holds for the first, most pronounced extremum in the ratio. With increasing $q$ the difference between the peak maximum in the autoionization spectrum and the position in the photoabsorption spectrum decreases.

Figure 12 shows the PCS for $q=-2.5$ and $q=-3.5$, which show a long tail on the low-energy side due to the negative Fano parameter. The ratio obtained is also similar to that obtained by two cross sections with positive effective Fano parameter, i.e. as long as both $q$-values show the same sign the resulting ratios are similar in the shape. Contrary to the ratios for positive $q$, the peak maxima of the Ar $1 s^{-1} 4 p$ resonance and the most pronounced extremum in the ratio are below the energy position of the $\mathrm{Ar} 1 s^{-1} 4 p$ resonance in the photoabsorption spectrum.

Figure 13 shows the PCSs and ratios based on effective Fano parameters with the same absolute value, but different sign. The associated ratios show more structure than in case of equal sign for $q$ since they consist of three extrema instead of two, as seen as seen in figures 11 and 12. But once again, the ratios for $|q|=2.5$ and $|q|=3.5$ are rather similar. In summary, the ratios allow us to distinguish between $q$ values of equal and opposite sign. This can also be seen in figure 7 , where representative ratios are shown. For each combination of $q$, i.e. two positive values (red solid line), one positive and one negative value (blue dashed line) and two negative values (black dash-dotted line) one representative ratio is shown. For all ratios the non-interacting cross section is assumed to be zero.

\section{ORCID iDs}

Ralph Püttner (i) https://orcid.org/0000-0002-8761-6873

Tatiana Marchenko (D) https://orcid.org/0000-0002-90153339

Loïc Journel (D) https://orcid.org/0000-0001-8044-5437
Maria Novella Piancastelli (D) https://orcid.org/0000-00033303-7494

Steven T Manson (D) https://orcid.org/0000-0002-7072-4122

\section{References}

[1] Rueff J-P et al 2015 J. Synchrotron Radiat. 22175

[2] Céolin D et al 2013 J. Electron Spectrosc. Relat. Phenom. 190 188

[3] Piancastelli M N, Marchenko T, Guillemin R, Journel L, Travnikova O, Ismail I and Simon M 2019 Rep. Prog. Phys. 83016401

[4] Walker T E H, Berkowitz J, Dehmer J L and Waber J T 1973 Phys. Rev. Lett. 31678

[5] Ron A, Kim Y S and Pratt R H 1981 Phys. Rev. A 241260

[6] Kim Y S, Pratt R H and Ron A 1981 Phys. Rev. A 241889

[7] Berkowitz J 1979 Photoabsorption, Photoionization and Photoelectron Spectroscopy (New York: Academic)

[8] Samson J A R 1982 Handbuch der Physik ed W Mehlhorn vol 31 (Berlin: Springer) pp 123-213

[9] Dias E W B et al 1997 Phys. Rev. Lett. 784553

[10] Drube W, Grehk T M, Thiess S, Pradhan G B, Varma H R, Deshmukh P C and Manson S T 2013 J. Phys. B: At. Mol. Opt. Phys. 46245006

[11] Keating D A, Manson S T, Dolmatov V K, Mandal A, Deshmukh P C, Naseem F and Kheifets A S 2018 Phys. Rev. A 98013420

[12] Breinig M, Chen M H, Ice G E, Parente F, Crasemann B and Brown G S 1980 Phys. Rev. A 22520

[13] Avaldi L, Dawber G, Camilloni R, King G C, Roper M, Siggel M R F, Stefani G and Zitnik M 1994 J. Phys. B: At. Mol. Opt. Phys. 273953

[14] Kramida A, Ralchenko Y, Reader J (NIST ASD Team) 2020 NIST Atomic Spectra Database (version 5.8) National Institute of Standards and Technology, Gaithersburg, MD https:// physics.nist.gov/asd

[15] Samson J A R 1976 Phys. Rep. 28 303-54

[16] Johnson W R and Lin C D 1979 Phys. Rev. A 20964

[17] Johnson W R, Lin C D, Cheng K T and Lee C M 1980 Phys. Scr. 21409

[18] Lee C M and Johnson W R 1980 Phys. Rev. A 22979

[19] Fano U 1961 Phys. Rev. 1241866

[20] Russek A and Mehlhorn W 1986 J. Phys. B: At. Mol. Phys. 19 911-27

[21] Guillemin R et al 2015 Phys. Rev. A 92012503

[22] Derevianko A, Johnson W R and Cheng K T 1999 At. Data Nucl. Data Tables 73153

[23] Starace A F 1977 Phys. Rev. A 16231

[24] Menzel A, Frigo S P, Whitfield S B, Caldwell C D and Krause M O 1996 Phys. Rev. A 542080

[25] Fano U and Cooper J W 1965 Phys. Rev. 137 A1364

[26] Bethe H A and Salpeter E E 1957 Quantum Mechanics of Oneand Two-Electron Atoms (Berlin: Springer) pp 63-71

[27] Dyall K G 1983 J. Phys. B: At. Mol. Phys. 163137

[28] Hansen D L et al 1999 Phys. Rev. A 60 R2641

[29] Chakraborty H S, Hansen D L, Hemmers O, Deshmukh P C, Focke P, Sellin I A, Heske C, Lindle D W and Manson S T 2001 Phys. Rev. A 63042708

[30] Starace A F 1982 Handbuch der Physik ed W Mehlhorn vol 31 (Berlin: Springer) pp 1-121

[31] Liu C-N and Starace A F 1999 Phys. Rev. A 59 R1731

[32] Chen M H, Crasemann B and Mark H 1981 Phys. Rev. A 24177 